\title{
Synthesis and Properties of Platinum Nanoparticles by Pulsed Laser Ablation in Liquid
}

\author{
Maria Isabel Mendivil Palma, ${ }^{1}$ Bindu Krishnan, ${ }^{1,2}$ Guadalupe Alan Castillo Rodriguez, \\ Tushar Kanti Das Roy, ${ }^{1}$ David Avellaneda Avellaneda, ${ }^{1}$ and Sadasivan Shajii ${ }^{1,2}$ \\ ${ }^{1}$ Facultad de Ingeniería Mecánica y Eléctrica, Universidad Autónoma de Nuevo León, 66455 San Nicolás de los Garza, NL, Mexico \\ ${ }^{2}$ CIIDIT, Universidad Autónoma de Nuevo León, 66600 Apodaca, NL, Mexico
}

Correspondence should be addressed to Sadasivan Shaji; sshajis@yahoo.com

Received 3 January 2016; Revised 11 March 2016; Accepted 31 March 2016

Academic Editor: Bhanu P. S. Chauhan

Copyright (C) 2016 Maria Isabel Mendivil Palma et al. This is an open access article distributed under the Creative Commons Attribution License, which permits unrestricted use, distribution, and reproduction in any medium, provided the original work is properly cited.

Platinum (Pt) nanoparticles were synthesized by pulsed laser ablation in liquid (PLAL) technique in different liquids (acetone, ethanol, and methanol). Ablation was performed using a Q-switched Nd:YAG laser with output energy of $230 \mathrm{~mJ} / \mathrm{pulse}$ for $532 \mathrm{~nm}$ wavelength. Ablation time and laser energy fluence were varied for all the liquids. Effects of laser energy fluence, ablation time, and nature of the liquid were reported. The mean size, size distributions, shape, elemental composition, and optical properties of Pt nanoparticles synthesized by PLAL were examined by transmission electron microscopy (TEM), X-ray photoelectron spectroscopy (XPS), and UV-Visible absorption spectroscopy.

\section{Introduction}

Colloidal noble metal nanoparticles (NPs) have shown size dependent optical properties as well as interesting properties of magnetism and catalysis. Platinum (Pt) and its alloy nanoparticles have attracted much attention because of their potential application in many catalytic reactions to eliminate nitrous oxide (NO) generated in combustion process [1]. There are various synthesis routes for the fabrication of $\mathrm{Pt}$ and Pt-compounds at nanoscale such as radiolysis, hydrogen reduction and citrate reduction [2], and pulsed laser ablation deposition [3]. Pulsed laser ablation in liquid is a rapid, simple, and versatile synthesis technique to obtain ultrapure noble metal nanoparticle colloids. By pulsed laser ablation in liquid, synthesis of Pt NPs [4-9] with different size, shapes, and properties has been reported by various research groups. Also the preparation of highly dispersed platinum nanoparticle-graphene nanosheet (PtNP-GNS) hybrid colloidal solution [10] and charged Pt-Ir alloy nanoparticles by femtosecond laser ablation in acetone [11] were reported. Excimer pulsed laser ablation of a platinum target in water resulted in platinum hollow micro-/nanoparticles under different laser fluence [8]. However, no studies are known on fabrication of Pt nanoparticles in methanol and acetone by this method. The effects of ablation time on Pt NPs production and morphologies are also of interest.

We reported synthesis of metal, semiconductor, and ceramic nanoparticles using pulsed laser ablation in liquid technique in which the shape, size, structure, composition, and optical properties were analyzed [12-18]. In this paper, we report the effects of liquid medium (acetone, ethanol, and methanol), ablation energy fluence, and ablation time on morphology, size, structure, composition, and optical properties of platinum nanoparticles produced by PLAL technique. These liquids selected have different properties such as refractive index, dipole moment, and viscosity that can influence the particle morphologies and size distributions.

\section{Materials and Methods}

Pulsed laser ablation in liquid (PLAL) experiments were performed using a Q-switched Nd:YAG laser (Solar Laser System 
LQ929A) with output energy of $230 \mathrm{~mJ} /$ pulse for $532 \mathrm{~nm}$ wavelength. The pulse width was $10 \mathrm{~ns}$ and the repetition rate was $10 \mathrm{~Hz}$ for this laser. The target was a metal plate of Pt with high purity (99.99\%). The liquids selected were acetone, ethanol, and methanol to study the effects of the liquid media. Ablation was done with three different laser energy fluence for each liquid selected. Due to the high flammability, the selected liquids were diluted with distilled water (DW) in a ratio of 4:1 (liquid:DW) in order to avoid the combustion by the highly intense pulsed laser beam. The Pt target was placed at the bottom of a glass beaker with $10 \mathrm{~mL}$ of the liquid (liquid layer height of $9 \mathrm{~mm}$ ). The laser output was focused using a convex lens of $20 \mathrm{~cm}$. To vary the energy fluence of pulsed laser ablation, the working distance lens to target (DLTT) was varied as 19,18 , and $17 \mathrm{~cm}$. The energy fluence calculated was 25,19 , and $9 \mathrm{~J} / \mathrm{cm}^{2}$, for an output energy of $230 \mathrm{~mJ} /$ pulse of $532 \mathrm{~nm}$ wavelength. Ablation experiments were carried out for 5 minutes for the three energy fluence in all three liquids. For the high energy fluence $\left(25 \mathrm{~J} / \mathrm{cm}^{2}\right)$, the time of ablation was varied to 10 and 15 minutes also. The clear solution became brown color after laser ablation. The ablation productivity was improved with a translation system attached to the beaker containing the target at a velocity of $50 \mu \mathrm{m} / \mathrm{s}$. This helped to avoid continuous irradiation at one point of the target. Soon after each experiment, a transmission electron microscope (TEM) grid was prepared by placing a drop of Pt nanocolloids on a Cu grid and drying it at ambient temperature. TEM analysis was carried out using a FEI Titan G2 80-300 TEM and elemental analysis using an Energy Dispersive X-ray Analyzer (EDAX) associated with TEM. The size distribution was obtained by measuring nanoparticle sizes from different TEM images taken from the same copper grid. To detect the elemental composition and chemical state of the ablated products, some drops of Pt nanocolloids were placed on a $\mathrm{Cu}$ tape and dried at ambient temperature for Xray photoelectron spectroscopy (XPS) analysis. XPS analysis was done using a Thermo Scientific K-Alpha XPS instrument with monochromatized $\mathrm{Al} \mathrm{K} \alpha$ radiation $(h \nu=1486.68 \mathrm{eV})$. All the spectra reported were recorded with reference to C $1 s$ peak $(284.6 \mathrm{eV})$. For optical property studies, UV-Visible absorption spectra measurements were carried out using a dual beam UV-Vis-NIR spectrometer (Shimadzu UV-1800) with quartz cells of $1 \mathrm{~cm}$ path length using the liquid medium at the reference and Pt nanocolloids at sample positions.

\section{Results and Discussion}

3.1. Morphology and Structure. The morphology and size distribution of Pt nanoparticles obtained by PLAL in acetone, ethanol, and methanol were characterized by TEM analysis such as Scanning Transmission Electron Microscopy (STEM), Bright Field (BF), and High Resolution Transmission Electron Microscopy (HRTEM). Figure 1 shows the micrographs of Pt nanoparticles obtained in acetone, ethanol, and methanol by ablation wavelength of $532 \mathrm{~nm}$ for 5 minutes under three different laser energy fluence $\left(9,19\right.$, and $\left.25 \mathrm{~J} / \mathrm{cm}^{2}\right)$. The Pt nanoparticle size distribution histograms and the average diameters are given as insets in each case. In acetone (Figures 1(a), 1(b), and 1(c)), spherical nanoparticles are obtained with an average size of $1.9 \pm 1.5 \mathrm{~nm}$ for $9 \mathrm{~J} / \mathrm{cm}^{2}$ and $2.4 \pm 1.1 \mathrm{~nm} 19 \mathrm{~J} / \mathrm{cm}^{2}$ (particle size ranges from 1 to $6 \mathrm{~nm}$ ) while for the highest energy fluence of $25 \mathrm{~J} / \mathrm{cm}^{2}$, it shows an average size of $4.6 \pm 1.7 \mathrm{~nm}$ with a size range $(2$ to $8 \mathrm{~nm}$ ) with bigger or agglomerated nanoparticles. For ablation of platinum target in ethanol under low energy fluence $\left(9 \mathrm{~J} / \mathrm{cm}^{2}\right)$, well dispersed spherical nanoparticles with average size of $2.1 \pm 1.1 \mathrm{~nm}$ (particle size ranges from 1 to $6 \mathrm{~nm}$ ) are obtained while an increase in energy fluence (19 and $25 \mathrm{~J} / \mathrm{cm}^{2}$ ) resulted in an increase in average diameter of the Pt nanoparticles as $4.3 \pm 1.3 \mathrm{~nm}$ and $3.7 \pm 1.5 \mathrm{~nm}$, respectively (particle size ranges from 2 to $7 \mathrm{~nm}$ ), maintaining the spherical shapes. In methanol, the average size of the platinum spherical nanoparticles shows a small increase from $2.3 \pm 1.4 \mathrm{~nm}$ (particle size ranges from 1 to $6 \mathrm{~nm}$ ) to $2.7 \pm 1.3 \mathrm{~nm}$ and $3.3 \pm 1.1 \mathrm{~nm}$ (particle size ranges from 2 to $7 \mathrm{~nm}$ ) for an increase in ablation energy from 9 to 19 and $25 \mathrm{~J} / \mathrm{cm}^{2}$. In general, for all three liquid media and energy fluence, spherical nanoparticles are obtained with the smaller size ranges for Pt nanoparticles. Also, some bigger nanoparticles are present for energy fluence of $25 \mathrm{~J} / \mathrm{cm}^{2}$ in acetone and ethanol while smaller nanoparticles are obtained in methanol for the same energy fluence.

To explore the effects of ablation time on the morphology and size of Pt nanoparticles, TEM analysis was carried out to those samples prepared at ablation times of 10 and 15 minutes. Figure 2 shows the micrographs corresponding to TEM analysis of Pt nanoparticles obtained under different ablation times keeping the laser energy fluence constant $\left(25 \mathrm{~J} / \mathrm{cm}^{2}\right)$. Corresponding size distributions of the nanoparticles are also shown in the inset of each figure. Figures 2(a)-2(c) show the morphologies and particle size distributions of $\mathrm{Pt}$ nanoparticles obtained in acetone for ablation times of 5 , 10 , and 15 minutes using $532 \mathrm{~nm}$ at $25 \mathrm{~J} / \mathrm{cm}^{2}$. In this case, as the ablation time increases, the average size of platinum nanoparticles decreases from $4.6 \pm 1.7 \mathrm{~nm}$ (particles of $2 \mathrm{~nm}$ to $8 \mathrm{~nm}$ in size) to $2.1 \pm 0.9$ and $2.8 \pm 1.3 \mathrm{~nm}$ (particles of 1 to $6 \mathrm{~nm}$ in size). Tiny platinum nanoparticles are present as observed in these figures while, for 15 minutes of ablation, these nanoparticles are getting grouped. Pt nanoparticles formed in ethanol show a decrease in their average size when the ablation time was increased from 5 to 15 minutes (Figures 2 (d) $-2(\mathrm{f})$ ). For 5 minutes of ablation, the average size is $3.7 \pm$ $1.5 \mathrm{~nm}$ (particles of 2-7 nm size) and for 10 and 15 minutes it is $2.3 \pm 0.7$ and $2.3 \pm 0.5 \mathrm{~nm}$, respectively (size range of $1-4 \mathrm{~nm}$ ). All these nanoparticles maintained their spherical shapes. Compared to Pt nanoparticles obtained in acetone, more agglomeration is observed in ethanol as well as in methanol (Figures 2(g)-2(i)). Spherical nanoparticles of average sizes $3.3 \pm 1.1 \mathrm{~nm}$ and $3.4 \pm 1$ (particle size varies from 2 to $6 \mathrm{~nm}$ ) are obtained for 5 and 10 minutes of ablation in methanol and $3.6 \pm 1.1 \mathrm{~nm}$ (size range from 2 to $7 \mathrm{~nm}$ ) for 15 minutes of ablation. Some bigger particles are also present for 15 minutes of ablation in methanol. As an effect of ablation time, a decrease in average size of the Pt nanoparticles is observed for ablation in acetone and ethanol while the same average size is maintained in methanol. Table 1 shows details of all 
$9 \mathrm{~J} / \mathrm{cm}^{2}$

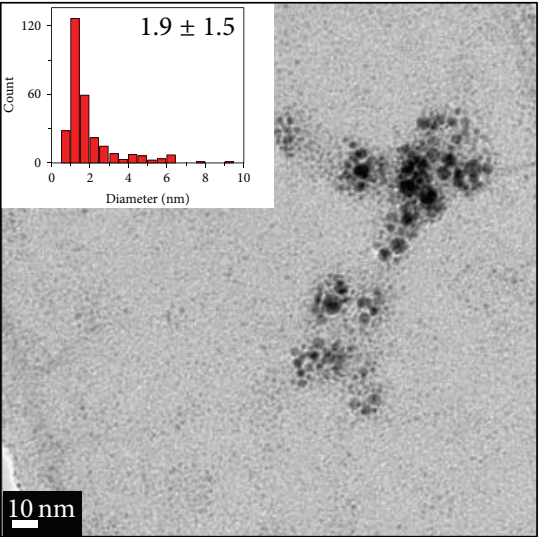

(a)

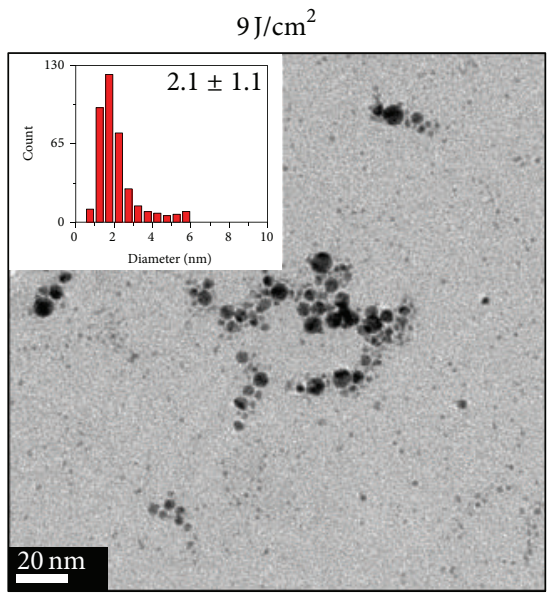

(d)

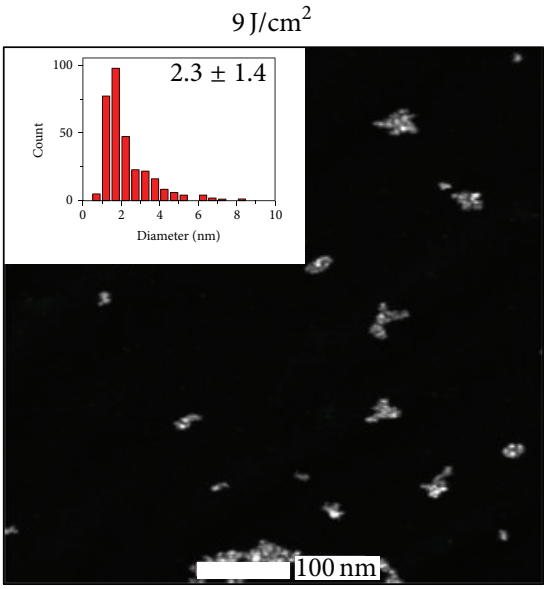

(g)
$19 \mathrm{~J} / \mathrm{cm}^{2}$

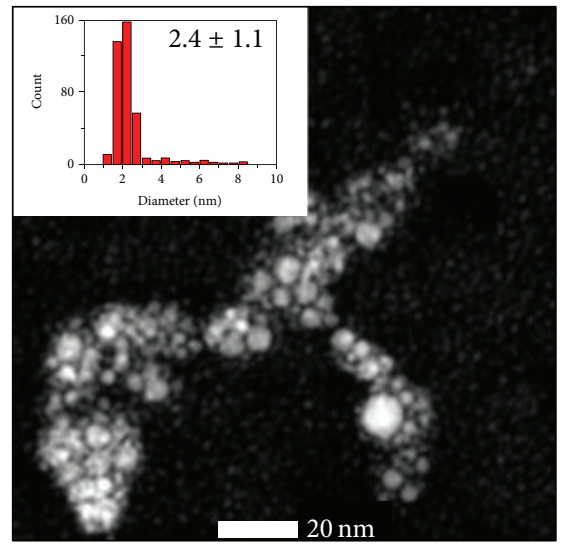

(b)

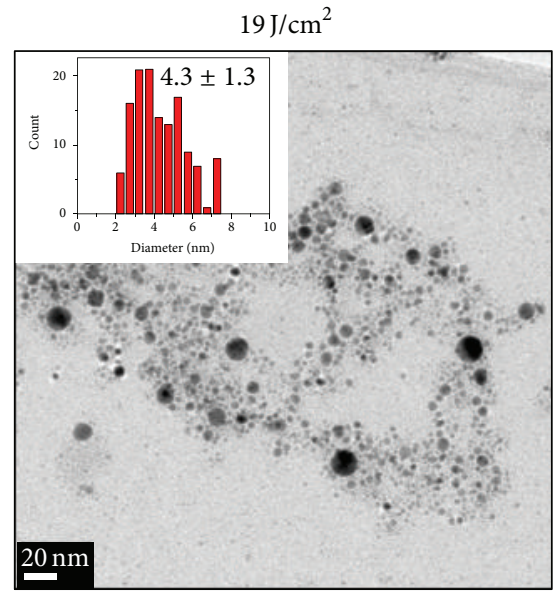

(e)

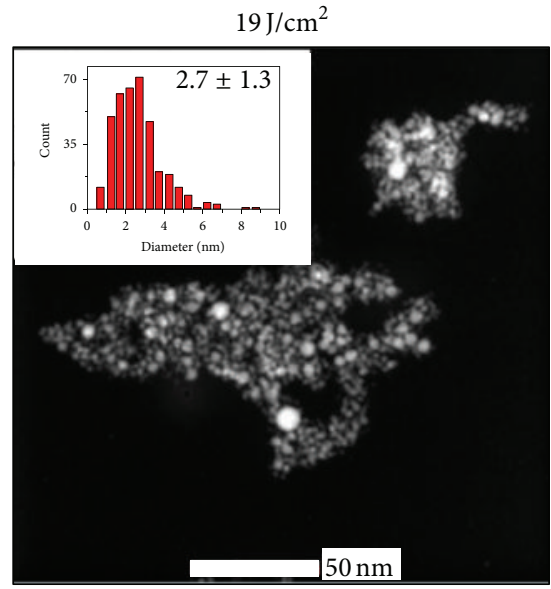

(h)
$25 \mathrm{~J} / \mathrm{cm}^{2}$

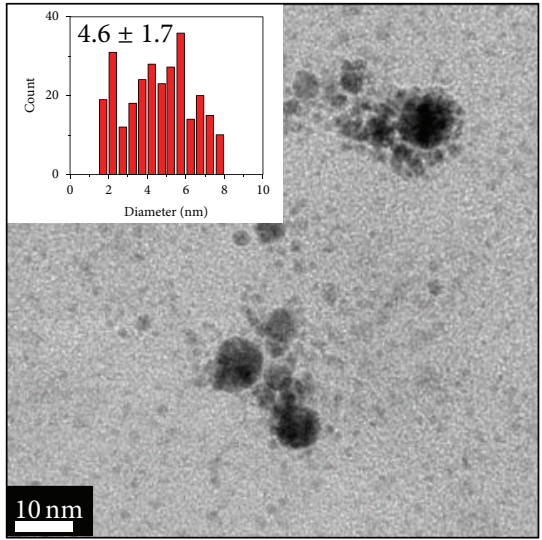

(c)

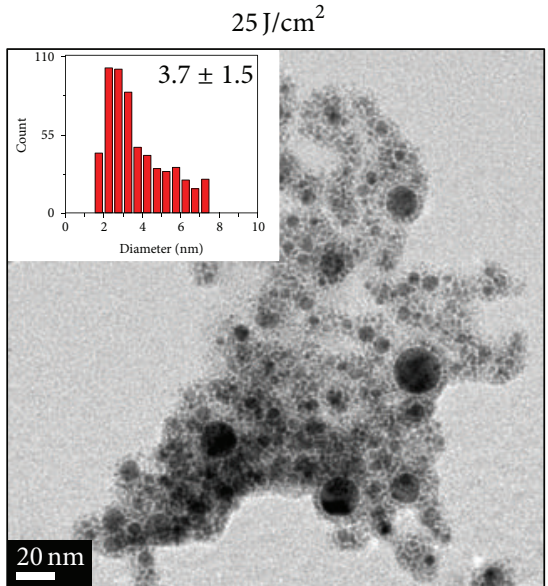

(f)

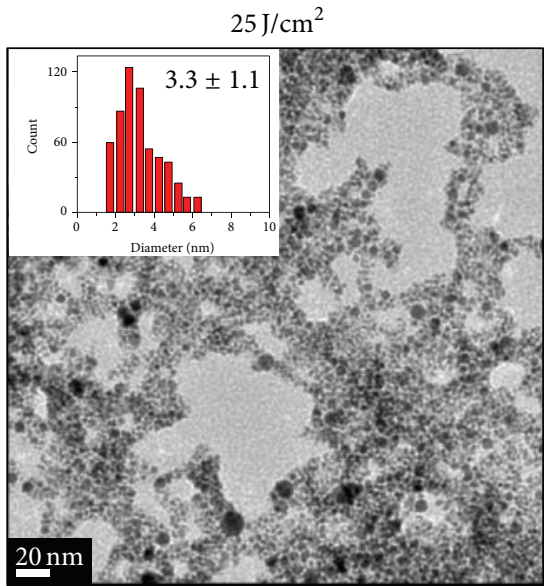

(i)

FIGURE 1: TEM micrographs and size distributions of Pt NPs obtained by PLAL in ((a)-(c)) acetone, ((d)-(f)) ethanol, and ((g)-(i)) methanol with 5 minutes of ablation and different energy fluence $\left(9,19\right.$, and $\left.25 \mathrm{~J} / \mathrm{cm}^{2}, 532 \mathrm{~nm}\right)$.

experimental conditions used to synthesize Pt nanoparticles such as liquid media, energy fluence, ablation time, number of particle counts (high and low) and their average sizes obtained in each case.
Ablation of Pt target in water using $532 \mathrm{~nm}(10 \mathrm{~Hz}$, $1.6 \mathrm{~J} / \mathrm{pulse} \mathrm{cm}^{2}$ ) resulted in nanoparticles of $4-7 \mathrm{~nm}$ with an average size $6.2 \pm 1.4 \mathrm{~nm}$ [4]. Similar synthesis (Pt NPs in water) using fundamental and harmonics (1064, 532 and 


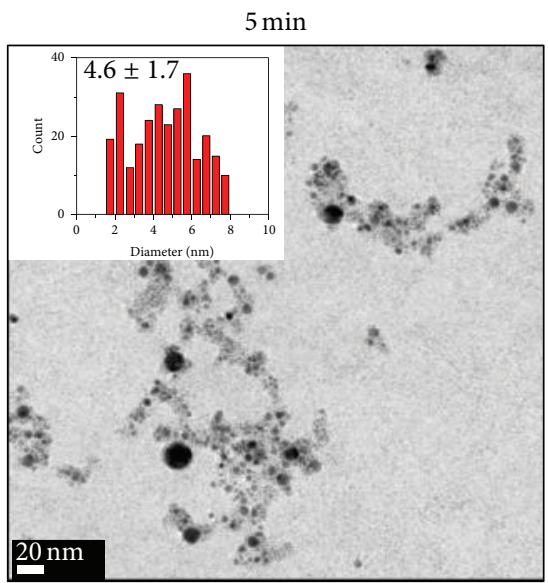

(a)

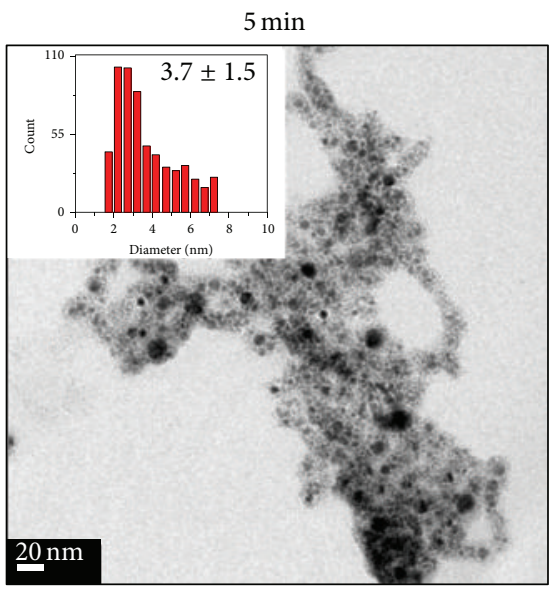

(d)

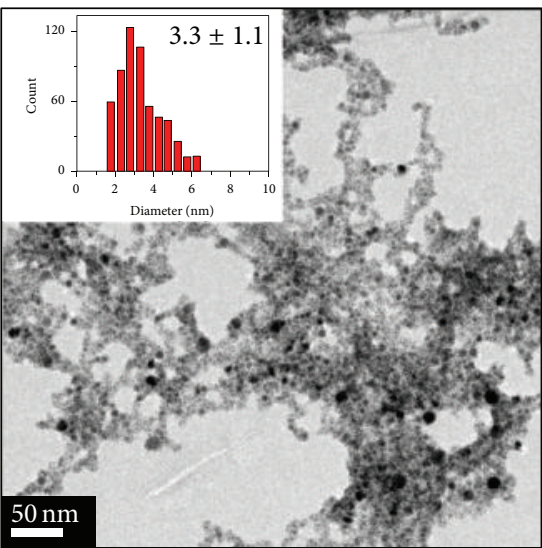

(g)

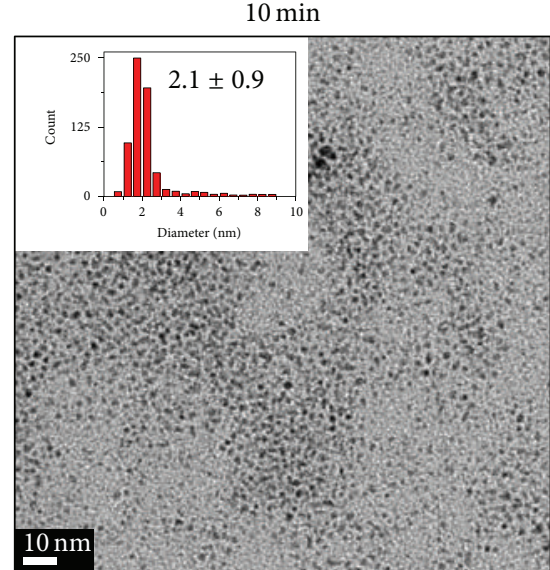

(b)

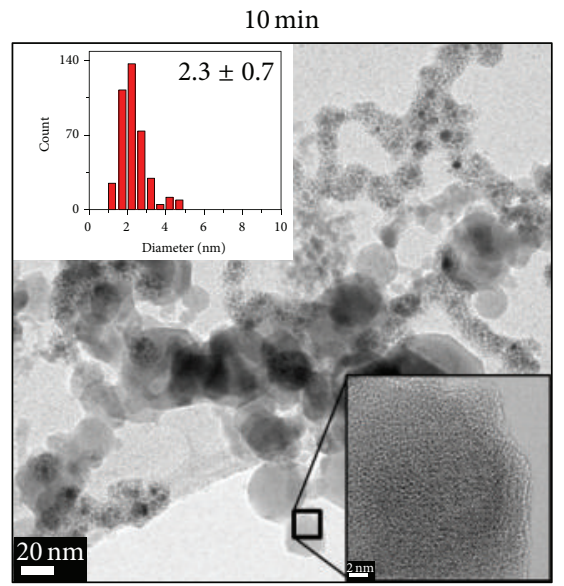

(e)

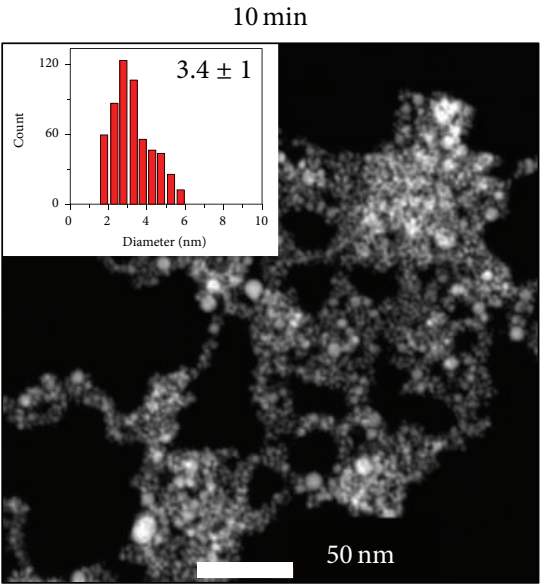

(h)

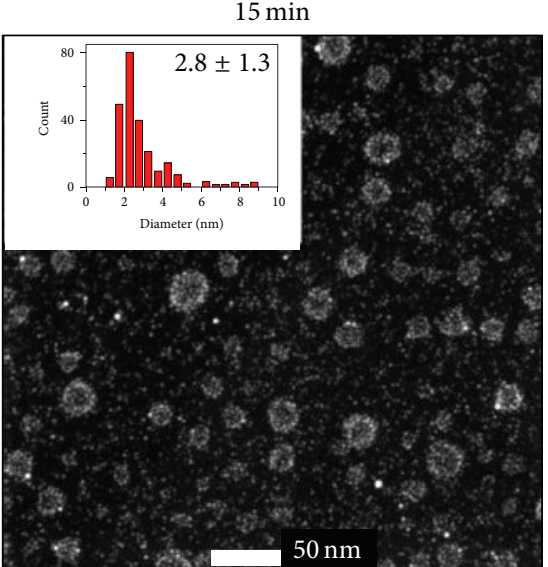

(c)

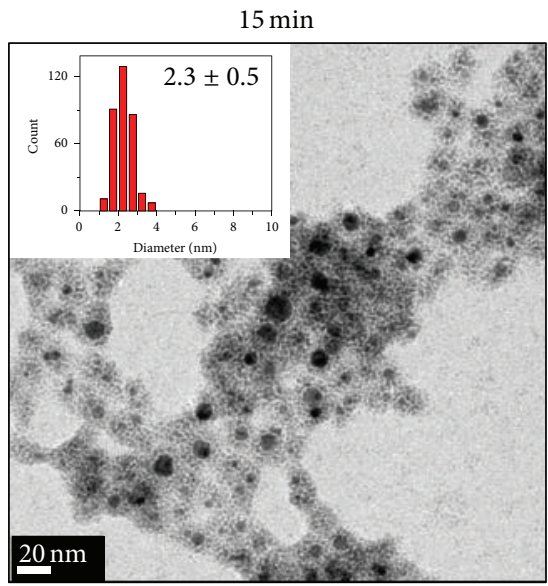

(f)

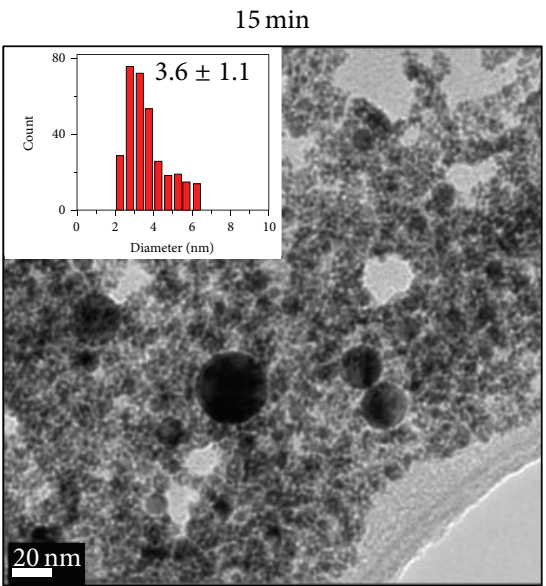

(i)

Figure 2: TEM micrographs and size distributions of Pt NPs obtained by PLAL in ((a)-(c)) acetone, ((d)-(f)) ethanol, and ((g) -(i)) methanol with $25 \mathrm{~J} / \mathrm{cm}^{2}$ and different ablation time $(5,10$, and 15 minutes, $532 \mathrm{~nm})$.

$355 \mathrm{~nm}$ ) of a nanosecond pulsed Nd:YAG laser resulted in average particle size of $3 \mathrm{~nm}$, while the infrared ablation resulted in a wider size distribution compared to other harmonics [6]. Excimer laser ablation ( $\mathrm{KrF}$ laser, $248 \mathrm{~nm}$, $10 \mathrm{~Hz}, 30 \mathrm{~ns}$ ) of a Pt target in water resulted in hollow micro-/nanoparticles [8]. Ablation of Pt target in ethanol using $1064 \mathrm{~nm}$ (Nd:YAG, average power $500 \mathrm{~mW}$, ablation time $15 \mathrm{~min}$ ) resulted in nanoparticles with a wide size range from $2 \mathrm{~nm}$ to $20 \mathrm{~nm}$ with an average of $9 \mathrm{~nm}$ [9]. In our study, we obtained Pt nanoparticles with much smaller size 
TABLE 1: Table lists details of experimental conditions such as liquid media, energy fluence, ablation time, number of particle counts (high and low), and their average sizes obtained.

\begin{tabular}{|c|c|c|c|c|c|c|}
\hline \multirow{2}{*}{$\begin{array}{l}\text { Condition } \\
\text { Liquid-fluence } \\
\left(\mathrm{J} / \mathrm{cm}^{2}\right) \text {-time }(\mathrm{min})\end{array}$} & \multicolumn{2}{|c|}{ Low counts } & \multicolumn{2}{|c|}{ High counts } & \multicolumn{2}{|c|}{ Final } \\
\hline & Size range $(\mathrm{nm})$ & Counts & Size range $(\mathrm{nm})$ & Counts & Number of particles & Average size $(\mathrm{nm})$ \\
\hline Acetone-9-5 & $2.75-3$ & 14 & $1.25-1.5$ & 126 & 291 & $1.9 \pm 1.5$ \\
\hline Acetone-19-5 & $1.25-1.5$ & 11 & $2.25-2.5$ & 157 & 401 & $2.4 \pm 1.1$ \\
\hline Acetone-25-5 & $7.25-8$ & 10 & $5.75-6$ & 36 & 291 & $4.6 \pm 1.7$ \\
\hline Acetone-25-10 & $3.25-3.5$ & 11 & $1.75-2$ & 251 & 636 & $2.1 \pm 0.9$ \\
\hline Acetone-25-15 & $4.25-4.5$ & 14 & $2.25-2.5$ & 80 & 237 & $2.8 \pm 1.3$ \\
\hline Ethanol-9-5 & $4.75-5$ & 6 & $1.75-2$ & 123 & 384 & $2.1 \pm 1.1$ \\
\hline Ethanol-19-5 & $2.25-2.5$ & 6 & $3.75-4$ & 21 & 133 & $4.3 \pm 1.3$ \\
\hline Ethanol-25-5 & $6.75-7$ & 17 & $2.25-2.5$ & 102 & 572 & $3.7 \pm 1.5$ \\
\hline Ethanol-25-10 & $3.75-4$ & 4 & $2.25-2.5$ & 138 & 406 & $2.3 \pm 0.7$ \\
\hline Ethanol-25-15 & $3.75-4$ & 7 & $2.25-2.5$ & 129 & 340 & $2.3 \pm 0.5$ \\
\hline Methanol-9-5 & $0.75-1$ & 5 & $1.75-2$ & 98 & 316 & $2.3 \pm 1.4$ \\
\hline Methanol-19-5 & $6.25-6.5$ & 4 & $2.75-3$ & 71 & 377 & $2.7 \pm 1.3$ \\
\hline Methanol-25-5 & $6.25-6.5$ & 13 & $2.75-3$ & 124 & 579 & $3.3 \pm 1.1$ \\
\hline Methanol-25-10 & $6-6.25$ & 7 & $2.75-3$ & 105 & 316 & $3.4 \pm 1$ \\
\hline Methanol-25-15 & $6.25-6.5$ & 14 & $2.75-3$ & 76 & 322 & $3.6 \pm 1.1$ \\
\hline
\end{tabular}

(2 to $3 \mathrm{~nm}$ ) than those of other reports on Pt NPs in water/ethanol. Also we analyzed the effect of ablation time on size of nanoparticles.

Figure 3 shows the HRTEM images and selected area electron diffraction (SAED) analysis of $\mathrm{Pt}$ nanoparticles. Figure 3(a) shows the HRTEM analysis with the crystalline structure of $\mathrm{Pt}$ nanoparticles in acetone obtained at $9 \mathrm{~J} / \mathrm{cm}^{2}$ for 5 minutes. The interplanar distance measured of $2.2 \AA$ from this HRTEM corresponds to (111) plane of face centered cubic (FCC) structure of platinum. SAED analysis (Figure 3(b), $9 \mathrm{~J} / \mathrm{cm}^{2}, 5 \mathrm{~min}$, acetone) shows the planes (111), (200), (220), (311), and (422) that are indexed with the PDF Number 040802 for FCC structure of Pt NPs. Figures 3(c) and 3(e) show the HRTEM micrographs corresponding to $2.2 \AA$, (111) plane of Pt FCC structure for the platinum nanoparticles obtained in ethanol $\left(25 \mathrm{~J} / \mathrm{cm}^{2}, 5 \mathrm{~min}\right)$ and methanol $\left(19 \mathrm{~J} / \mathrm{cm}^{2}, 5 \mathrm{~min}\right)$, respectively. Figures $3(\mathrm{~d})$ and $3(\mathrm{f})$ show the SAED analysis of Pt nanoparticles obtained in ethanol and methanol, respectively, under the same conditions as in the case of HRTEM. All these reflections and planes identified in SAED pattern analysis are in agreement with PDF Number 040802 for FCC structure of Pt.

Analysis of the structure of the platinum nanoparticles using HRTEM and SAED resulted in the fact that the nanoparticles maintained the FCC structure under different conditions. Thermal evaporation is the ablation mechanism related to pulsed laser ablation of Pt target in liquids using a nanosecond pulsed laser. During pulsed laser ablation in liquid, the laser beam wavelength and liquid medium influence the kinetics of nucleation and growth of nanoparticles leading to the formation of nanoparticles with different morphology, size, and structure. For a given target material and laser parameters, particle generation depends on the refractive index of the liquid medium at a given laser wavelength. Lower reflectivity of laser light at the solid-liquid interface favors higher rate of ablation, while absorption of laser light by the liquid reduces ablation rate. Also, the growth processes could be influenced by the physical properties of the liquid medium and then nanoparticles with different morphologies, size, and structure can result. It is reported that highly polar molecules tend to form electrical double layers on the surface of the as-prepared nanoparticles, preventing their growth, aggregation, and precipitation. Ablation time and energy fluence can affect the productivity of nanoparticles and the continuous ablation of nanoparticles in the colloids can end with laser fragmentation to finer nanoparticles or melting of the nanoparticles to bigger ones depending on the energy and ablation wavelength $[19,20]$.

In general, fabrication of nanoparticles depends on laser parameters, target material, and the nature of the liquid. Polarity, viscosity, and refractive index of the liquid at the ablation laser wavelength are important in determining the final size and size distribution [19, 20]. Values of dipole moment, absolute viscosity, and refractive index for acetone are $2.69 \mathrm{D}, 0.000316 \mathrm{Ns} / \mathrm{m}^{2}$, and 1.3614 for $532 \mathrm{~nm}$, respectively. Ethanol has these constants of $1.66 \mathrm{D}$, $0.001095 \mathrm{Ns} / \mathrm{m}^{2}$, and 1.3635 for $532 \mathrm{~nm}$ and for methanol $2.87 \mathrm{D}, 0.00056 \mathrm{Ns} / \mathrm{m}^{2}$, and 1.3288 for $532 \mathrm{~nm}$, respectively. Higher viscosity and dielectric constant resulted in well dispersed Pt nanoparticles with average sizes in the same range in methanol for different laser fluence and ablation time. In ethanol and acetone, the average size of Pt NPs increased with increase in ablation fluence. An increase in ablation time resulted in smaller nanoparticles in acetone 


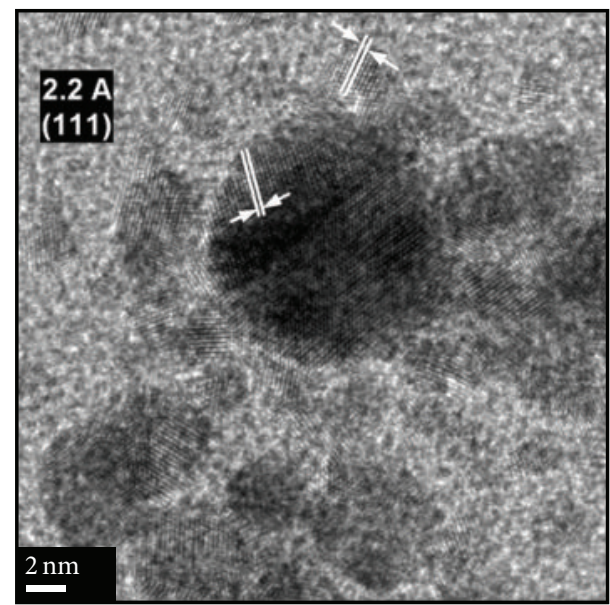

(a)

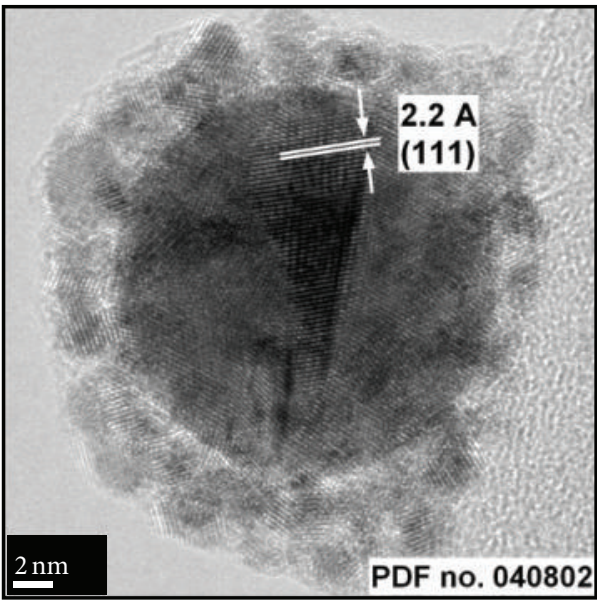

(c)

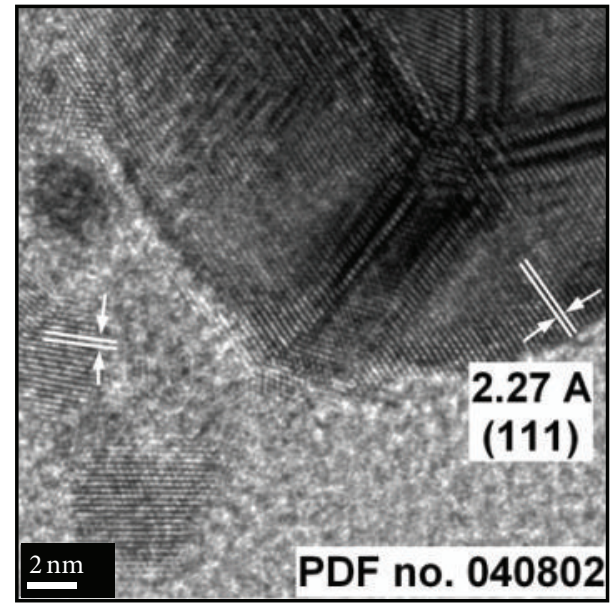

(e)

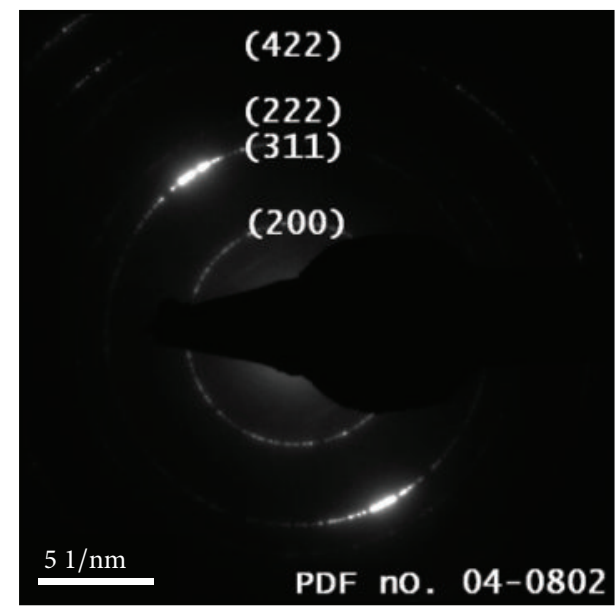

(b)

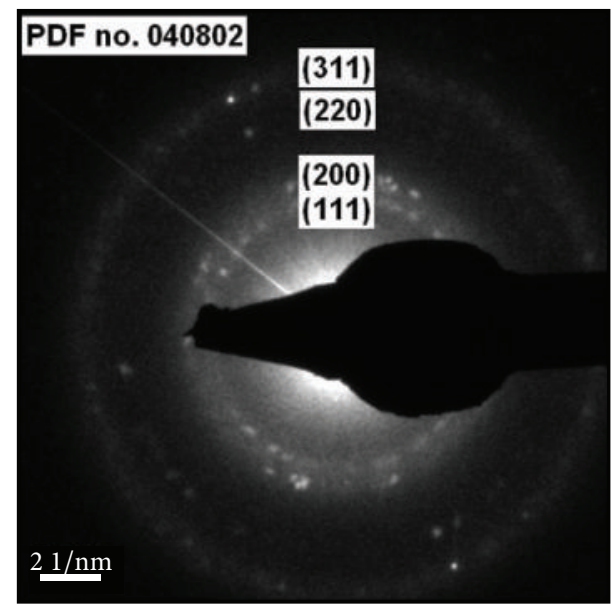

(d)

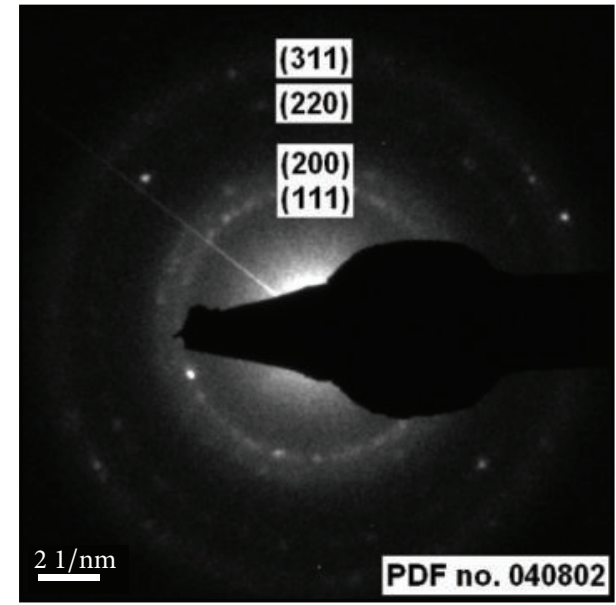

(f)

Figure 3: HRTEM and SAED micrographs of Pt NPs in ((a)-(b)) acetone (9 J/ $\mathrm{cm}^{2}, 5$ minutes) ((c)-(d)) ethanol $\left(25 \mathrm{~J} / \mathrm{cm}^{2}, 5 \mathrm{minutes}\right)$, and ((e)-(f)) methanol $\left(19 \mathrm{~J} / \mathrm{cm}^{2}, 5\right.$ minutes), respectively. 

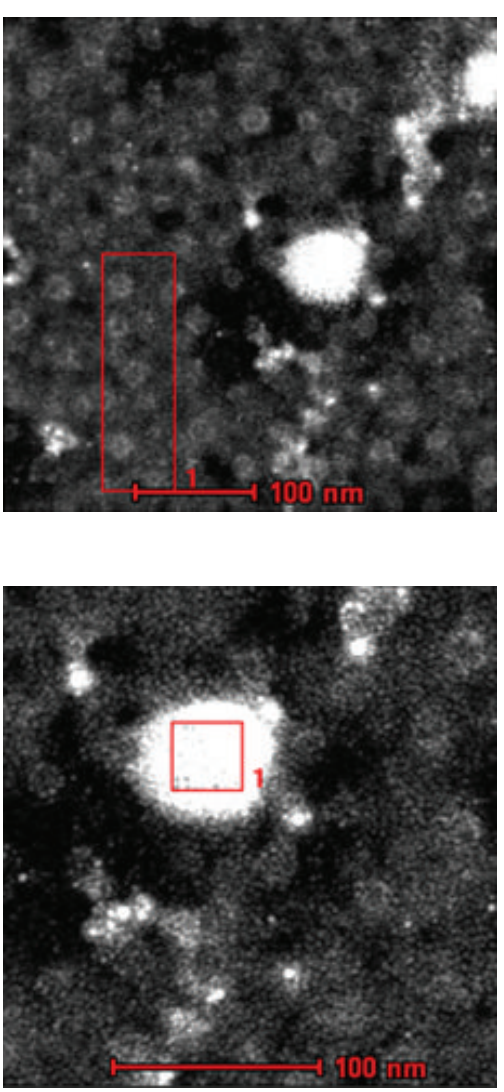

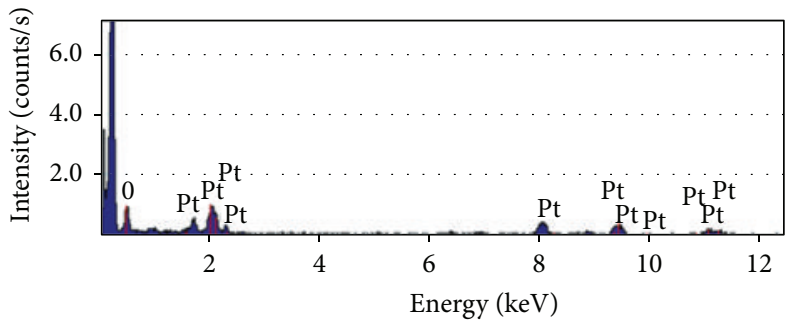

(a)

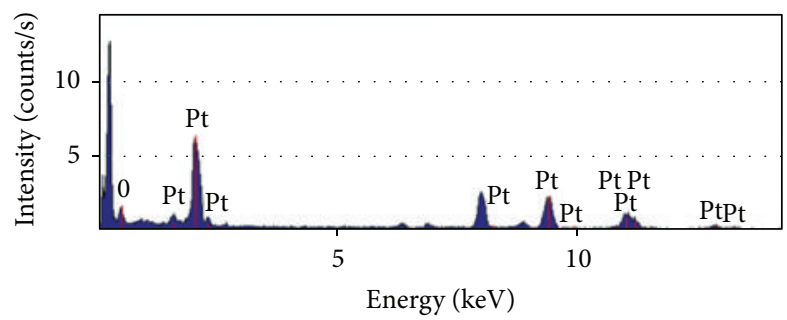

(b)

Figure 4: EDX spectrum of Pt NPs obtained by PLAL in acetone at (a) $19 \mathrm{~J} / \mathrm{cm}^{2}$ for 5 minutes of ablation and (b) $25 \mathrm{~J} / \mathrm{cm}^{2}$ for $15 \mathrm{minutes}$ of ablation over smaller nanoparticles and agglomerated NPs.

and ethanol. The decrease in size at higher ablation time can be due to the effect of continuous irradiation of particles in the colloidal solution at a high laser energy fluence $[19,20]$. For 5 minutes of ablation at lower fluence $\left(9 \mathrm{~J} / \mathrm{cm}^{2}\right)$, Pt NPs obtained in all these media showed nearly the same average size $(2 \pm 1 \mathrm{~nm})$, but there was an increase in average size with increase in fluence for all the three liquid media. Increase in energy fluence could result in increase in average size of nanoparticles as reported in many cases $[12,16,19,20]$. The effects of ablation time (at constant energy fluence of $25 \mathrm{~J} / \mathrm{cm}^{2}$ ) were different in these liquids. In acetone and ethanol, there was a decrease in average size with increase in ablation time. A small increase in average size was observed for Pt NPs in methanol (3.3 to $3.6 \mathrm{~nm}$ ). These changes could be due to the different properties of these liquids, mainly difference in dipole moment and viscosity [19].

3.2. Elemental Composition and Chemical State. Elemental composition of $\mathrm{Pt}$ nanoparticles synthesized by PLAL in different liquid media was characterized by EDX detector associated with TEM and X-ray photoelectron spectroscopy. In the case of EDX characterization, the samples obtained at $19 \mathrm{~J} / \mathrm{cm}^{2}$ for 5 minutes and $25 \mathrm{~J} / \mathrm{cm}^{2}$ for 15 minutes in acetone are analyzed (Figure 4). STEM micrographs with their corresponding EDX spectra are shown in Figure 4(a) at
$19 \mathrm{~J} / \mathrm{cm}^{2}$ for 5 minutes in acetone over smaller NPs groups and (b) shows a big agglomerated nanoparticle of Pt at $25 \mathrm{~J} / \mathrm{cm}^{2}$ for 15 minutes in acetone. In both EDX spectra, signals for $\mathrm{Pt}$ and a signal for oxygen with less contribution in the spectrum over the big agglomerated particle probably due to the high density of the NPs in comparison with smaller NPs are identified.

Pt nanoparticles obtained by PLAL in acetone, ethanol, and methanol were analyzed by XPS. The results obtained were very similar for all the liquid media (Figure 5). Figure 5(a) shows the XPS spectra of Pt NPs in acetone (5 minutes, $19 \mathrm{~J} / \mathrm{cm}^{2}$ ), in which the high resolution photoelectron peaks $4 \mathrm{f}_{7 / 2}$ and $4 \mathrm{f}_{5 / 2}$ at 72.02 and $75.22 \mathrm{eV}$, respectively, correspond to $\mathrm{Pt}^{0}[3,21,22]$. Figures 5(b) and 5(c) show the spectrum of Pt NPs in ethanol and methanol, respectively. From the figure, the peaks at binding energies of 71.22 and $70.86 \mathrm{eV}$ in ethanol and methanol, respectively, for $\mathrm{Pt} 4 \mathrm{f}_{7 / 2}$ are assigned to $\mathrm{Pt}^{0}$.

In this ablation process of platinum target in different liquid media, the nucleation of the particles occurs at the active mixing zone between the plasma plume created during ablation process and the confining liquid, and then the particles grow to their final size and shape in the liquid medium. For materials such as platinum, silver, and gold, which are not particularly reactive metal nuclei, they start 


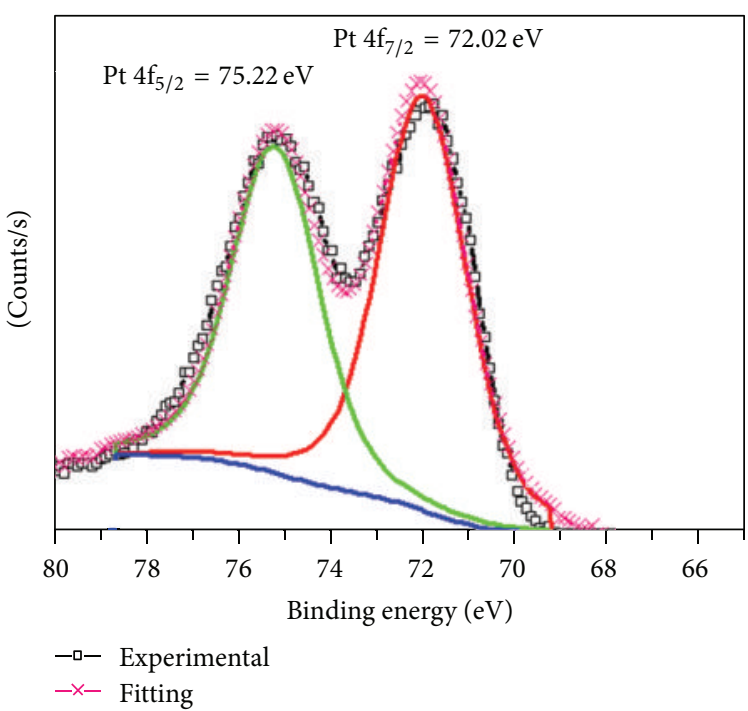

(a)

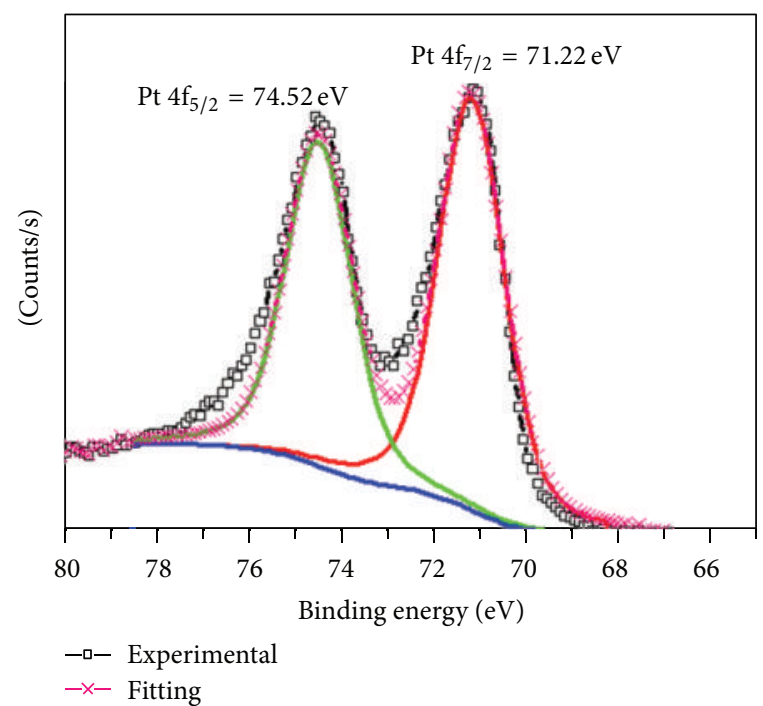

(b)

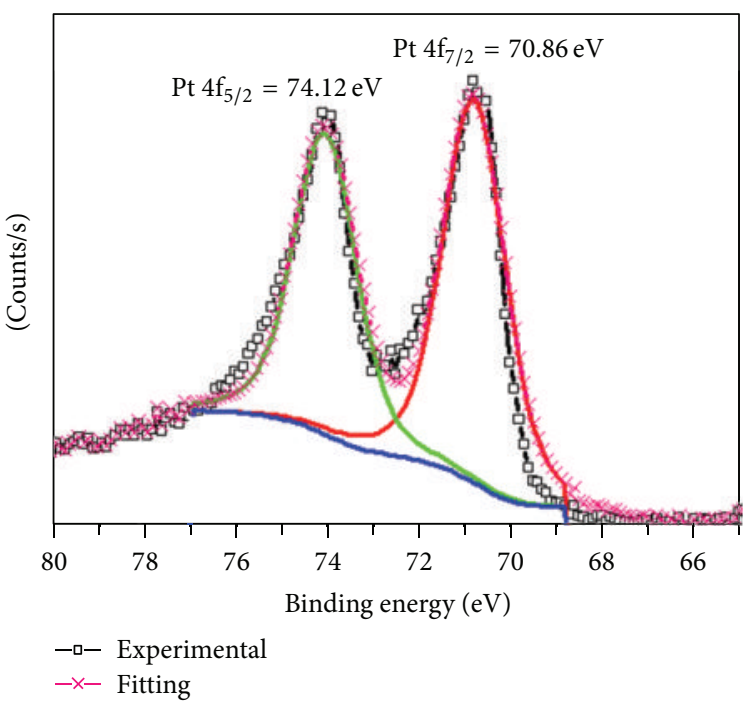

(c)

FIgURE 5: XPS spectrum of $4 \mathrm{f}$ core level of Pt NPs in (a) acetone, (b) ethanol, and (c) methanol (19 J/ $\mathrm{cm}^{2}$ for 5 minutes).

nucleation nonreactively and subsequently grow into metal nanoparticles. Moreover, the chemical composition of the liquid medium influences the reactions between the ablated species and the surrounding liquid defining the ablation is reactive type or nonreactive one. Because of the nonreactive nature of ablation of $\mathrm{Pt}$ target in these liquids, the nanoparticles obtained were in their elemental state [7].

3.3. Optical Properties. Optical properties of Pt nanocolloids synthesized by PLAL were investigated by UV-Visible absorption spectroscopy. Optical absorption spectra were measured immediately after experiments for each liquid media. The optical absorption spectra for Pt nanocolloids obtained under different ablation conditions of energy fluence and time are shown in Figure 6. Figures 6(a), 6(c), and 6(e) show the absorption spectra of Pt nanoparticles obtained in acetone, ethanol, and methanol, respectively, for different laser fluence $\left(9,19\right.$, and $\left.25 \mathrm{~J} / \mathrm{cm}^{2}\right)$. The surface plasmon absorption for Pt nanoparticles observed is included in the inset with an expanded scale for the absorption peak identification. Absorption spectra for Pt nanocolloids prepared under different ablation times (5, 10, and 15 minutes) for an energy fluence of $25 \mathrm{~J} / \mathrm{cm}^{2}$ are shown in Figures 6(b), 6(d), and 6(e), respectively, for acetone, ethanol, and methanol. The spectra of as-prepared Pt nanocolloids in acetone (Figure 6(a)) displayed a broad absorption band with the maximum intensity around $328-335 \mathrm{~nm}$ (inset of Figure 6(a)) with a decrease in intensity from the peaks towards UV as well as the visible region under both conditions of energy fluence and ablation time. The maximum absorption intensity (related to higher concentration of NPs) appeared from the lower energy fluence. This result is related to the increase in the spot area of 


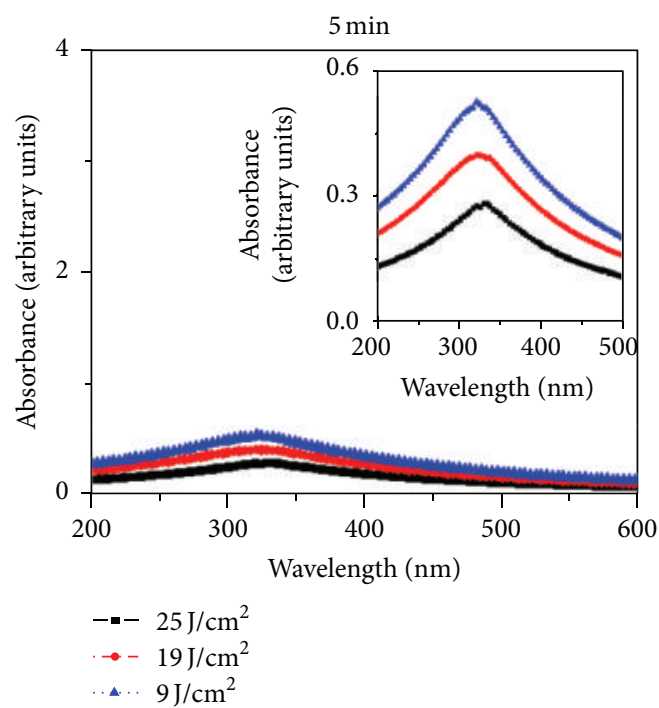

(a)

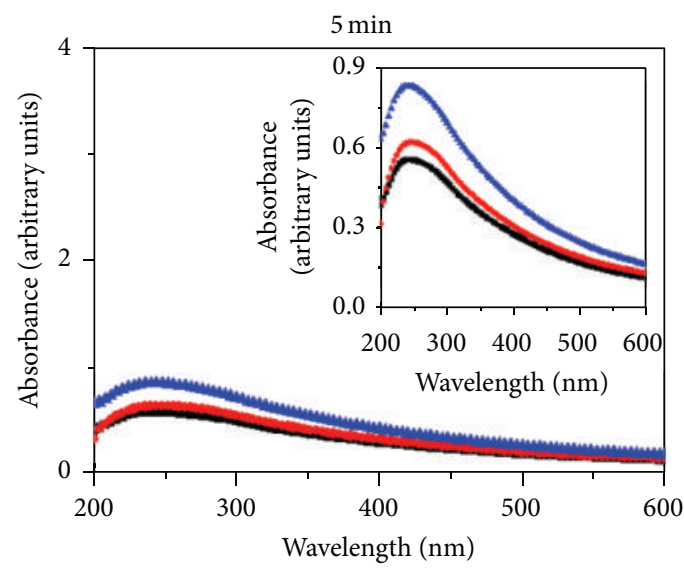

$--25 \mathrm{~J} / \mathrm{cm}^{2}$

$-\bullet-19 \mathrm{~J} / \mathrm{cm}^{2}$

$-\Delta-9 \mathrm{~J} / \mathrm{cm}^{2}$

(c)

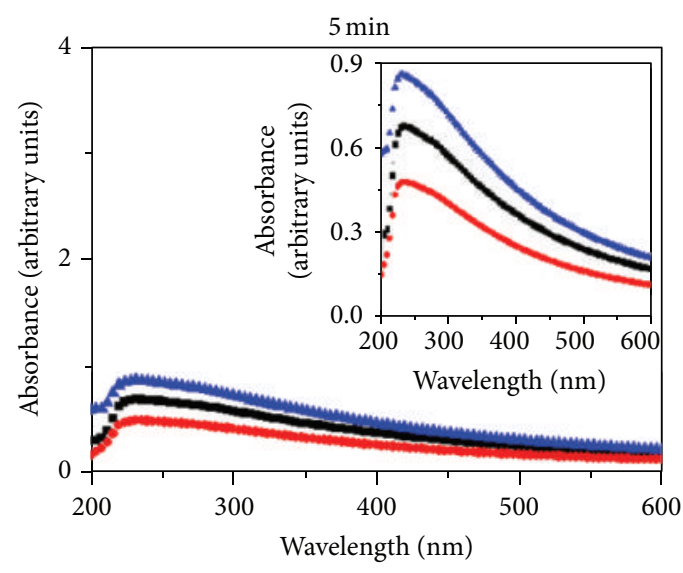

$--25 \mathrm{~J} / \mathrm{cm}^{2}$
$--19 \mathrm{~J} / \mathrm{cm}^{2}$
$-\Delta-9 \mathrm{~J} / \mathrm{cm}^{2}$

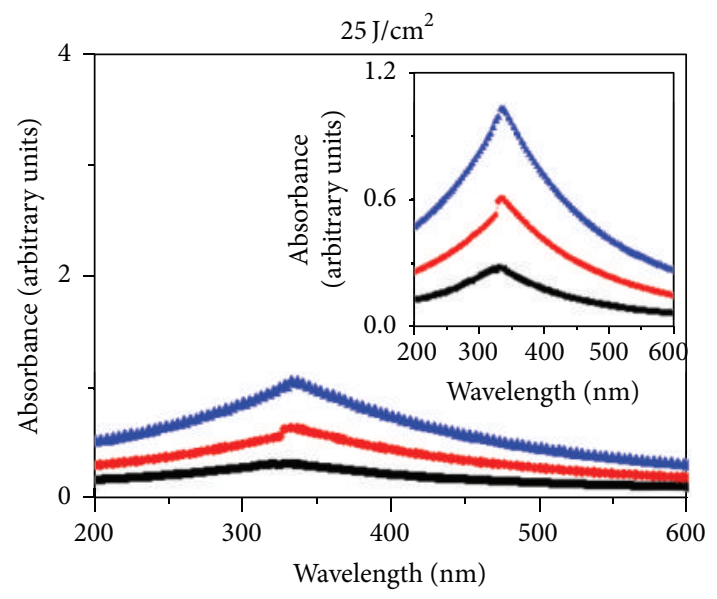

$--5 \mathrm{~min}$

- - $10 \mathrm{~min}$

$-\mathbf{-}-15 \mathrm{~min}$

(b)

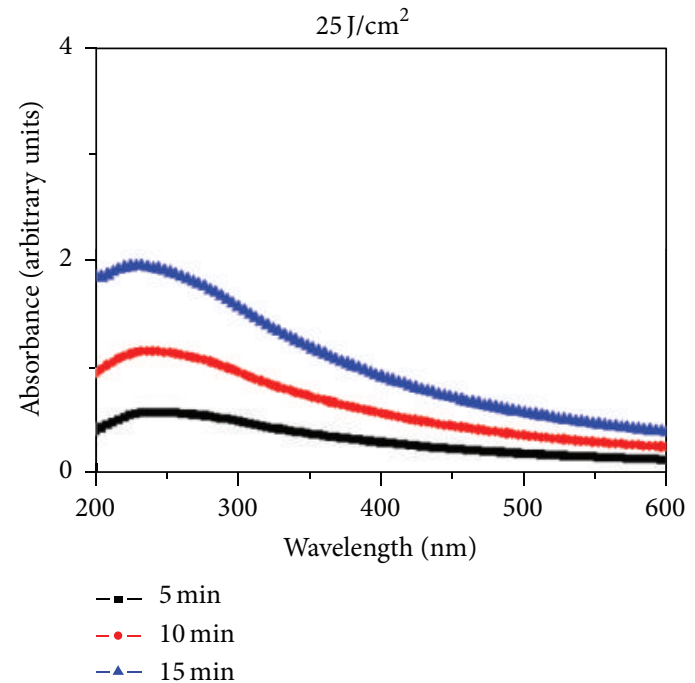

(d)

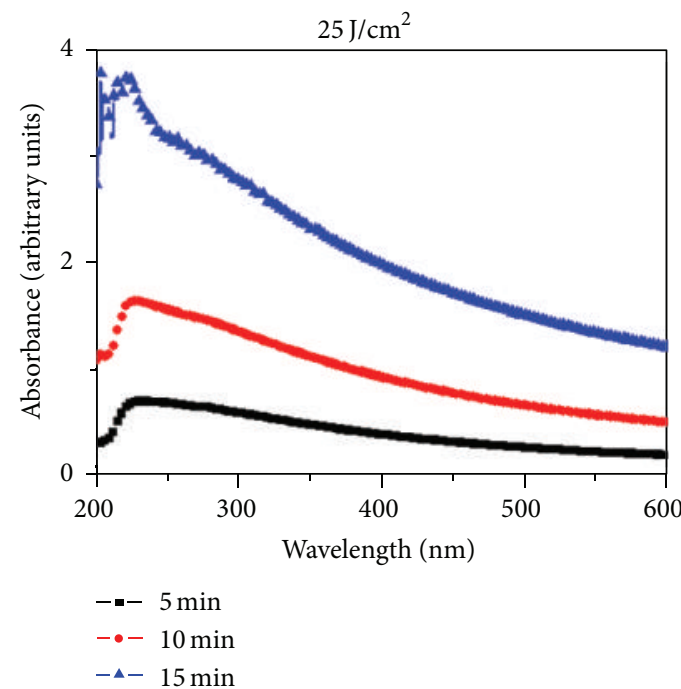

(f)

FIGURE 6: UV-Visible absorption spectra of Pt NPs obtained in ((a), (b)) acetone, ((c), (d)) ethanol, and ((e), (f)) methanol with different energy fluence and ablation time. Spectra with an expanded scale is shown as inset for (a), (b), (c), and (e). 
ablation. Figure 6(d) shows the optical absorption spectrum of Pt NPs obtained in acetone at $25 \mathrm{~J} / \mathrm{cm}^{2}$ for 5,10 , and 15 minutes of PLAL. As expected, higher absorption intensity corresponds to higher time of ablation, three times higher absorbance for 15 minutes of ablation in comparison with that of Pt NPs obtained by 5 minutes of ablation. The peak appeared at 329, 332, and $334 \mathrm{~nm}$ (inset of Figure 6(b)) for 5, 10 , and 15 minutes, respectively, the little red shift presented in the spectra could be due to an aggregation effect of the higher concentration of Pt NPs.

Figure 6(c) corresponds to absorption spectra of asprepared Pt NPs in ethanol at different energy fluence for 5 minutes of ablation. The UV-Vis absorption spectra present a broad peak with the maximum absorption intensity at 241, 246, and $242 \mathrm{~nm}$ (inset of Figure 6(c)) for 9, 19, and $25 \mathrm{~J} / \mathrm{cm}^{2}$, respectively. Similar to Pt NPs in acetone, the higher absorption intensity is obtained at lower energy fluence. Figure 6(d) shows the as-prepared Pt NPs in ethanol at $25 \mathrm{~J} / \mathrm{cm}^{2}$ for different time of ablation. The spectra present a broad peak at 242, 238, and $230 \mathrm{~nm}$ for 5, 10, and 15 minutes, respectively, showing a blue shift contrary to the results obtained in acetone, in which the increase in the ablation time induced a red shift in the spectrum. This blue shift in absorption peaks could be due to the presence of many tiny Pt nanoparticles and a broader spectral absorption nature for Pt nanocolloids in ethanol could be due to their agglomerated zones and presence of bigger particles.

Figure 6(e) shows absorption spectrum for Pt NPs in methanol at different energy fluence. The spectral nature is similar to that obtained in acetone, but with the maximum intensity at 231, 234, and $233 \mathrm{~nm}$ (inset of Figure 6(e)) for 9,19 , and $25 \mathrm{~J} / \mathrm{cm}^{2}$, respectively. The maximum intensity absorption corresponds to lower energy fluence, same as the results obtained in other liquid media. Figure 6(f) shows Pt nanocolloids in methanol at $25 \mathrm{~J} / \mathrm{cm}^{2}$ with different time of ablation. The maximum intensity absorption is at 233,228 , and $223 \mathrm{~nm}$ for 5, 10, and 15 minutes, respectively, the same as spectral nature and peak positions for that obtained at different energy fluence. Pt nanoparticles obtained in methanol under both conditions presented same kind of morphologies and size distributions that might have contributed to the same kind of optical spectral response.

For all the liquid media, the higher absorbance is for the Pt nanocolloids synthesized at lower energy fluence for ablation time of 5 minutes because of the larger spot area of irradiation. Also the highest absorbance is for longer ablation time as expected due to higher concentration of $\mathrm{Pt}$ nanoparticles. As an effect of the liquid medium, the $\mathrm{Pt}$ nanoparticles obtained in acetone, ethanol, and methanol presented different absorption peak values and spectral response. According to the literature $[4,5]$ and theoretical calculations [23], Pt spherical nanoparticles of around $10 \mathrm{~nm}$ presented an optical band in the far UV $(<215 \mathrm{~nm}$ in water) and was attributed to interband transitions. In our case, the peak appeared at around $330 \mathrm{~nm}$, which is in agreement with the results reported by You et al. [24] that presented spectra with size dependence peaks in this range for Pt NPs of 4$8 \mathrm{~nm}$. The absorption spectra were in agreement with that of Pt nanoparticles prepared by PLAL as well as chemical methods. Pt NPs obtained in acetone showed better stability (up to 7 weeks) compared to those in ethanol and methanol. Further studies are needed on long term stability of these Pt nanocolloids.

\section{Conclusions}

Pt nanoparticles were synthesized in acetone, ethanol, and methanol by PLAL technique under different ablation energy fluence and time. TEM analysis showed spherical morphologies and cubic crystalline structures for the Pt nanoparticles. Their elemental composition and chemical states were confirmed using EDX and XPS analysis. The variation of energy fluence and ablation time influenced the size and morphologies of the Pt NPs obtained in different liquids. Optical properties of the Pt nanocolloids were different for different liquid media while the highest concentrations of nanoparticles were for lowest energy fluence and longer ablation time.

\section{Competing Interests}

The authors declare that there are no competing interests regarding the publication of this paper.

\section{Acknowledgments}

The authors are thankful to SEP-CONACYT-Mexico (Project no. 214282), PROMEP-Mexico, and PAICYT-UANL, Mexico, for the financial assistance. Maria Isabel Mendivil Palma is grateful to CONACYT-Mexico for providing a research fellowship.

\section{References}

[1] P. Ascarelli, V. Contini, and R. Giorgi, "Formation process of nanocrystalline materials from $\mathrm{x}$-ray diffraction profile analysis: application to platinum catalysts," Journal of Applied Physics, vol. 91, no. 7, pp. 4556-4561, 2002.

[2] A. Henglein, B. G. Ershov, and M. Malow, "Absorption spectrum and some chemical reactions of colloidal platinum in aqueous solution," Journal of Physical Chemistry, vol. 99, no. 38, pp. 14129-14136, 1995.

[3] P. Bommersbach, M. Chaker, M. Mohamedi, and D. Guay, "Physico-chemical and electrochemical properties of platinumtin nanoparticles synthesized by pulsed laser ablation for ethanol oxidation," The Journal of Physical Chemistry C, vol. 112, no. 37, pp. 14672-14681, 2008.

[4] F. Mafuné, J.-Y. Kohno, Y. Takeda, and T. Kondow, "Formation of stable platinum nanoparticles by laser ablation in water," The Journal of Physical Chemistry B, vol. 107, no. 18, pp. 4218-4223, 2003.

[5] W. T. Nichols, T. Sasaki, and N. Koshizaki, "Laser ablation of a platinum target in water. I. Ablation mechanisms," Journal of Applied Physics, vol. 100, no. 11, Article ID 114911, 2006.

[6] W. T. Nichols, T. Sasaki, and N. Koshizaki, "Laser ablation of a platinum target in water. II. Ablation rate and nanoparticle size distributions," Journal of Applied Physics, vol. 100, no. 11, Article ID 114912, 2006. 
[7] W. T. Nichols, T. Sasaki, and N. Koshizaki, "Laser ablation of a platinum target in water. III. Laser-induced reactions," Journal of Applied Physics, vol. 100, no. 11, Article ID 114913, 2006.

[8] Z. Yan, R. Bao, and D. B. Chrisey, "Excimer laser ablation of a Pt target in water: the observation of hollow particles," Nanotechnology, vol. 21, no. 14, Article ID 145609, 2010.

[9] T. B. Nguyen, T. D. Nguyen, Q. D. Nguyen, and T. T. Nguyen, "Preparation of platinum nanoparticles in liquids by laser ablation method," Advances in Natural Sciences: Nanoscience and Nanotechnology, vol. 5, no. 3, Article ID 035011, 2014.

[10] Y. Yang, M. Shi, Q.-F. Zhou, Y.-S. Li, and Z.-W. Fu, "Platinum nanoparticle-graphene hybrids synthesized by liquid phase pulsed laser ablation as cathode catalysts for Li-air batteries," Electrochemistry Communications, vol. 20, no. 1, pp. 11-14, 2012.

[11] J. Jakobi, A. Menéndez-Manjón, V. S. K. Chakravadhanula, L. Kienle, P. Wagener, and S. Barcikowski, "Stoichiometry of alloy nanoparticles from laser ablation of PtIr in acetone and their electrophoretic deposition on PtIr electrodes," Nanotechnology, vol. 22, no. 14, Article ID 145601, 2011.

[12] M. I. Mendivil, B. Krishnan, G. A. Castillo, and S. Shaji, "Synthesis and properties of palladium nanoparticles by pulsed laser ablation in liquid," Applied Surface Science, vol. 348, pp. 45-53, 2015.

[13] M. I. Mendivil, B. Krishnan, F. A. Sanchez et al., "Synthesis of silver nanoparticles and antimony oxide nanocrystals by pulsed laser ablation in liquid media," Applied Physics A: Materials Science and Processing, vol. 110, no. 4, pp. 809-816, 2013.

[14] M. I. Mendivil, L. V. García, B. Krishnan, D. Avellaneda, J. A. Martinez, and S. Shaji, "CuInGaSe ${ }_{2}$ nanoparticles by pulsed laser ablation in liquid medium," Materials Research Bulletin, vol. 72, pp. 106-115, 2015.

[15] M. I. Mendivil, S. Shaji, G. A. Castillo, and B. Krishnan, "Transmission electron microscopic studies on noble metal nanoparticles synthesized by pulsed laser ablation in liquid," in Microscopy: Advances in Scientific Research and Education, A. Méndez-Vilas, Ed., pp. 911-920, Formatex Research Center, Badajoz, Spain, 2014.

[16] G. A. Castillo Rodriguez, G. G. Guillen, M. I. Mendivil Palma et al., "Synthesis and characterization of hercynite nanoparticles by pulsed laser ablation in liquid technique," International Journal of Applied Ceramic Technology, vol. 12, no. 2, pp. E34E43, 2015 .

[17] D. Garza, G. Grisel García, M. I. Mendivil Palma et al., "Nanoparticles of antimony sulfide by pulsed laser ablation in liquid media," Journal of Materials Science, vol. 48, no. 18, pp. 6445-6453, 2013.

[18] G. G. Guillén, M. I. M. Palma, B. Krishnan et al., "Structure and morphologies of $\mathrm{ZnO}$ nanoparticles synthesized by pulsed laser ablation in liquid: effects of temperature and energy fluence," Materials Chemistry and Physics, vol. 162, pp. 561-570, 2015.

[19] H. Zeng, X.-W. Du, S. C. Singh et al., "Nanomaterials via laser ablation/irradiation in liquid: a review," Advanced Functional Materials, vol. 22, no. 7, pp. 1333-1353, 2012.

[20] S. V. Rao, G. K. Podagatlapalli, and S. Hamad, "Ultrafast laser ablation in liquids for nanomaterials and applications," Journal of Nanoscience and Nanotechnology, vol. 14, no. 2, pp. 1364-1388, 2014.

[21] J. B. Yadav, J.-W. Park, Y.-J. Cho, and O.-S. Joo, "Intermediate hydroxide enforced electrodeposited platinum film for hydrogen evolution reaction," International Journal of Hydrogen Energy, vol. 35, no. 19, pp. 10067-10072, 2010.
[22] M. Martin-Martinez, A. Álvarez-Montero, L. M. GómezSainero et al., "Deactivation behavior of $\mathrm{Pd} / \mathrm{C}$ and $\mathrm{Pt} / \mathrm{C}$ catalysts in the gas-phase hydrodechlorination of chloromethanes: structure-reactivity relationship," Applied Catalysis B: Environmental, vol. 162, pp. 532-543, 2015.

[23] J. A. Creighton and D. G. Eadon, "Ultraviolet-visible absorption spectra of the colloidal metallic elements," Journal of the Chemical Society, Faraday Transactions, vol. 87, no. 24, pp. 38813891, 1991.

[24] J. B. You, X. W. Zhang, J. J. Dong et al., "Localized-surfaceplasmon enhanced the $357 \mathrm{~nm}$ forward emission from $\mathrm{ZnMgO}$ films capped by Pt nanoparticles," Nanoscale Research Letters, vol. 4, no. 10, pp. 1121-1125, 2009. 

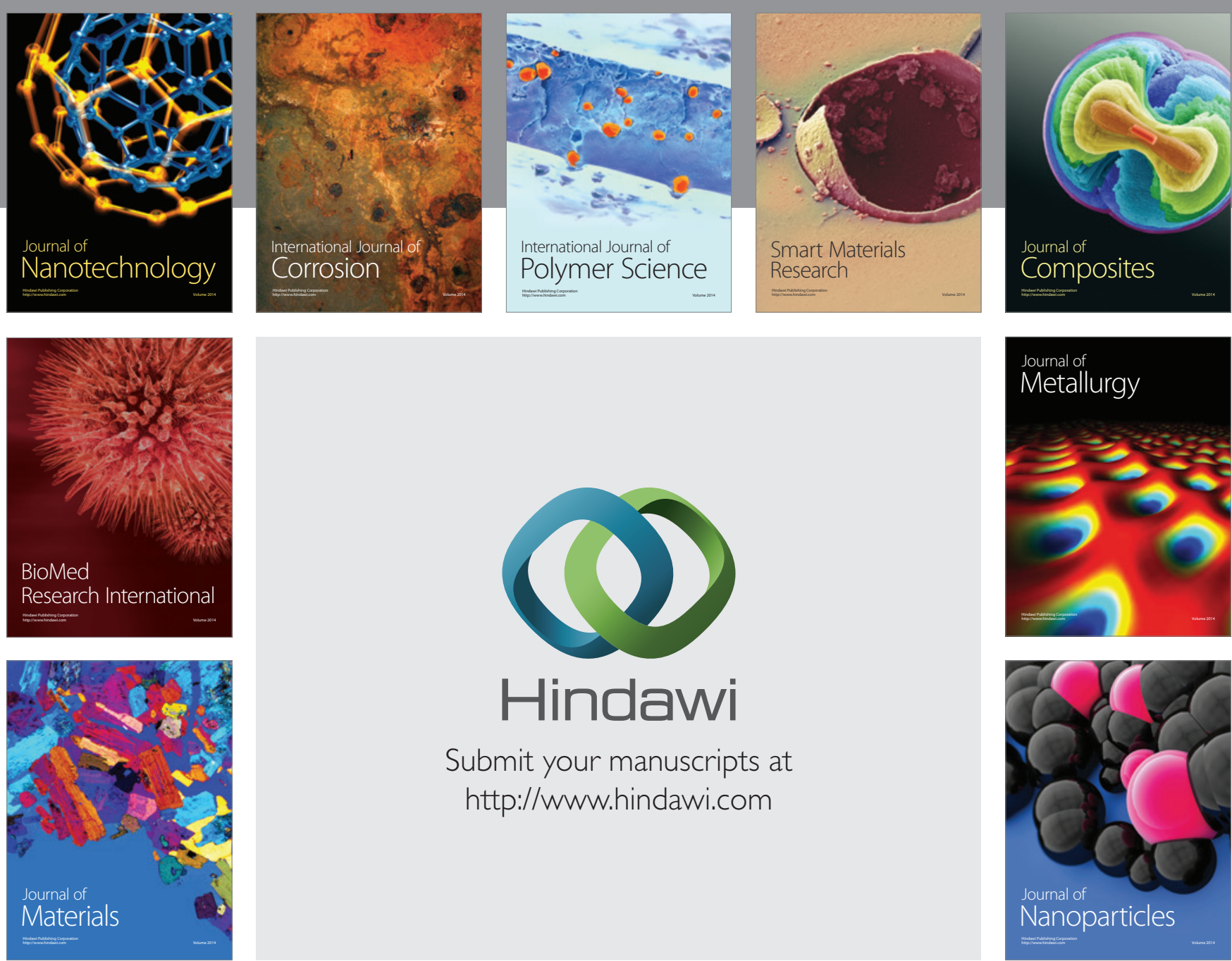

\section{Hindawi}

Submit your manuscripts at

http://www.hindawi.com

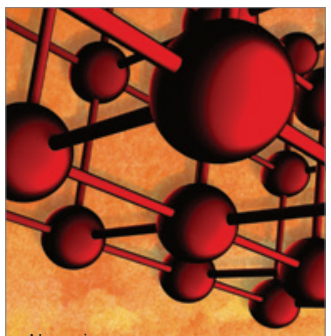

Materials Science and Engineering
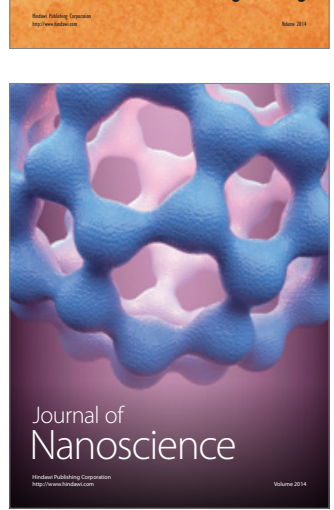
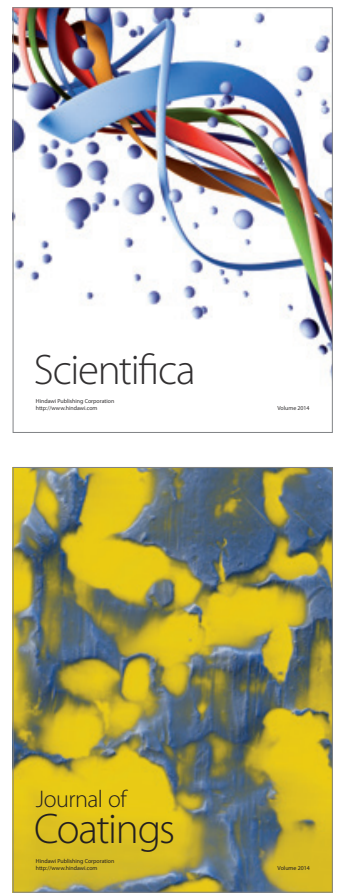
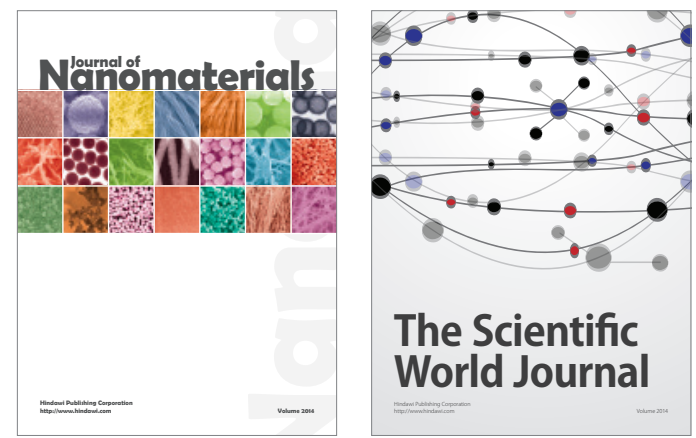

The Scientific World Journal
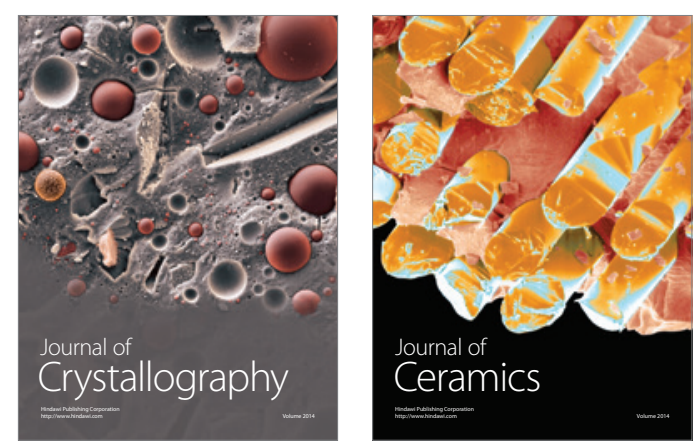
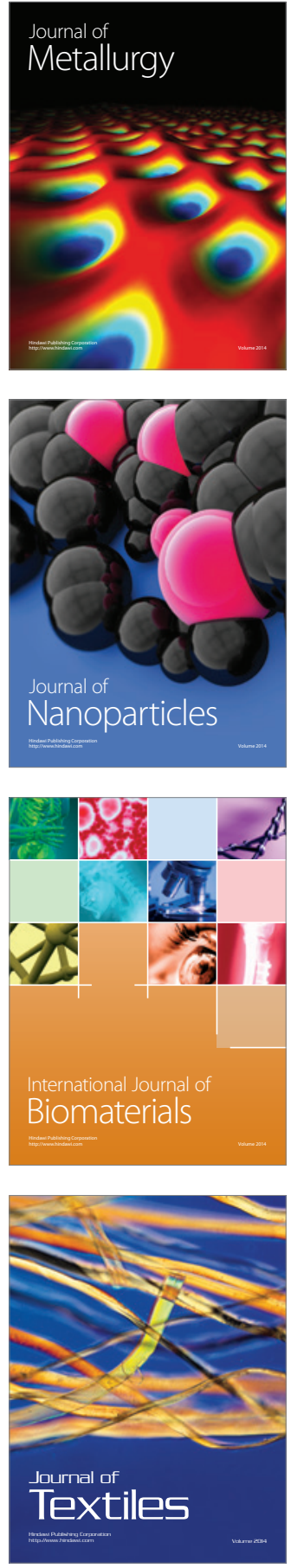